\title{
Les minéraux lourds des sables du littoral de la Guyane française : bilan sur l'origine des dépôts de la plate-forme des Guyanes
}

\author{
Michel PUJOS*, Jean-Claude PONS, Mario PARRA
}

UMR EPOC 5805-CNRS, département de géologie et océanographie, université Bordeaux 1, avenue des Facultés, 33405 Talence cedex, France

Reçu le 11 mai 1999 ; révisé le 8 décembre 1999 ; accepté le 2 février 2000

Résumé - Les minéraux lourds des sables de plage et des cordons littoraux de la Guyane française sont assemblés en trois cortèges: amphiboles dominantes, staurotides, épidotes et pyroxènes dans la presqu'île de Cayenne; staurotides cardinales, tourmalines et amphiboles jusqu'à la Mana; grenats, staurotides et tourmalines de la Mana au Maroni. En mer, les cortèges minéralogiques des sables offrent une grande similitude et une grande continuité avec les précédents de par leur composition minéralogique et leur répartition géographique. Il existe une parfaite cohérence entre ces cortèges de minéraux lourds et ceux décrits dans les fleuves et les roches mères qui constituent le bouclier guyanais. Les arguments mis en exergue montrent que les minéraux lourds et les sables qui les renferment sont issus d'apports locaux. (C) 2001 Ifremer/CNRS/IRD/Éditions scientifiques et médicales Elsevier SAS

\begin{abstract}
Heavy minerals in sediments of the French Guiana coast: sources of deposits on Guiana shelves. The distribution of heavy minerals on beaches and beach ridges sands of French Guiana shows three distinct suites from East to West: amphibole (largely dominant), staurolite, epidote and pyroxene off the Cayenne region, tourmaline and amphibole mainly off the Kourou-Mana, garnet, staurolite and tourmaline off the Mana-Maroni. A very good continuity exists between the assemblages of heavy minerals from the catchment area parent-rocks, the fluviatile sediments, the coastal zone and the shelf. The results of this study indicate and strengthen a local origin for the sandy sediments and do not support a possibility of an Amazonian source. (C) 2001 Ifremer/CNRS/IRD/Éditions scientifiques et médicales Elsevier SAS
\end{abstract}

\section{Guyane française / plages / cordons sableux / minéraux lourds}

\section{French Guiana / beaches / beach ridges sands / heavy minerals}

\section{INTRODUCTION}

Depuis le XIX $\mathrm{X}^{\mathrm{e}}$ siècle, les côtes de la Guyane française ont fait l'objet de campagnes menées par le Service hydrographique et océanographique de la Marine

*Correspondance et tirés à part.

Adresse e-mail : m.pujos@geocean.u-bordeaux.fr (M. PUJOS).
(S.H.O.M.) dans le but de réaliser des relevés hydrographiques (Yayer, 1948). Quelques dragages et prélèvements de roches ont été effectués au cours de ces missions avant que ne débute réellement la reconnaissance sédimentologique et géologique du littoral par l'étude des dépôts fins d'origine amazonienne qui bordent le littoral septentrional de l'Amérique du Sud, de l'embouchure du fleuve à la mer Caraïbe (Eisma et Van der Marel, 1971). Ce prisme côtier a très vite attiré l'attention de nombreux chercheurs : évolution du système dispersif amazonien, 
progradation et évolution côtière ont fait l'objet d'études régionales à l'instigation des pays riverains (Brésil, Surinam et Guyana).

L'étude des plages et des cordons littoraux est beaucoup plus fragmentaire que celle des vases littorales malgré la présence des minéraux lourds qui sont d'excellents traceurs de l'origine des dépôts (Imbrie et Van Andel, 1964 ; Pomerol, 1968 ; Lapierre, 1970 ; Jones et Davies, 1979).

Dans les années 1970 l'école hollandaise effectue des recherches sur le Surinam :

- Augustinus (1978) puis Augustinus et al. (1989) identifient deux provinces sédimentaires sur les côtes du Surinam, chacune constituant « une unité d'âge, d'origine et de distribution ». Pour ces auteurs, les sables à staurotide proviennent du fleuve Maroni et des côtes de la Guyane française alors que le cortège des sables à épidote-hornblende est probablement originaire de l'Amazone. Les sables grossiers à staurotide, grenat, tourmaline et zircon, les sables moyens à staurotide dominante, épidote et hornblende, et les sables fins à épidote et hornblende dominantes montrent des relations entre espèces minérales et classes granulométriques ;

- Krook $(1969$; 1979 ; 1988 ; 1992) décrit deux cortèges minéralogiques sur les mêmes côtes du Surinam, l'un à staurotide dominante dans les cordons littoraux et les plages, l'autre à staurotide-épidote dans les sables fins.

Ces auteurs observent une décroissance du grain moyen des sables et des teneurs en minéraux lourds d'est en ouest. Le changement graduel se manifeste sur les espèces minérales avec une augmentation de l'épidote et de la hornblende et une diminution concomitante de la staurotide et du grenat. Ces travaux montrent qu'une partie des sables fins - et de leur contenu minéralogique (épidotehornblende) - dérive de l'Amazone, transportée en suspension avec les argiles. D'autres espèces minérales (zircon, tourmaline, rutile) seraient issues de l'arrière pays (bouclier guyanais).

Peu de travaux concernent les plages et les cordons de la Guyane française.

- Bouysse et al. (1977) effectuent une reconnaissance sommaire. Sur la plage de Montabo (presqu'île de Cayenne) les minéraux opaques sont prépondérants, dominés par la magnétite (60 à 90\%), l'ilménite $(<10 \%)$ et la limonite $(<10 \%)$. Le cortège est complété par des zircons, staurotides, amphiboles et épidotes, tous de teneurs inférieures à $1 \%$. Sur la plage des Roches
(Kourou) la staurotide est cardinale (60 à 90\%) associée à de l'ilménite $(<10 \%)$ et du zircon $(<10 \%)$.

- Prost (1989; 1992) confirme l'abondance des minéraux opaques. Les sables noirs de la plage de Rémire (presqu'île de Cayenne) sont presque exclusivement composés d'ilménite, de magnétite et de gœtite (93\%) ainsi que de zircons et d'épidotes d'origine locale. Sur la plage Galibi (haut estran de la pointe Isère) la staurotide est cardinale (93\%) associée à des grenats, tourmaline et ilménite.

Ce travail présente la distribution des différentes espèces minérales rencontrées sur les plages et les cordons, puis compare ces cortèges et ceux précédemment reconnus dans les fleuves et sur la plate-forme continentale (Pujos et al., 1990). Les relations entre provinces réceptrices (côtes, plateau continental de la Guyane française) et distributrices (bouclier guyanais, bassin versant de l'Amazone) apportent un témoignage sur l'origine des dépôts.

\section{SITES ET MÉTHODES D'ÉCHANTILLONNAGE}

Les plaines côtières de la Guyane française présentent deux ensembles situés respectivement à l'ouest et à l'est de Cayenne: à l'ouest, les plages sont jalonnées de cordons sableux de plus en plus nombreux à l'approche du Surinam. À l'est, les plaines en sont dépourvues.

- Du Maroni à la rivière de Cayenne alternent: a) des plages et des cordons sableux bordés à l'intérieur des terres par des marais et des savanes marécageuses, séparées les unes des autres par d'anciens cordons sableux ; b) des mangroves ininterrompues, la frange littorale ne présentant aucun intermédiaire morphologique entre continent et océan.

- Le littoral de la presqu'île de Cayenne est constitué d'anses sableuses dont l'avant-plage est, à l'instar des autres plages de la Guyane, périodiquement envahi par des vases qui se déplacent le long du littoral vers l'WNW (Froidefond et al., 1988).

- De Cayenne à l'Oyapock, le paysage littoral subit un profond changement. La mangrove borde l'océan et s'étend sur de grandes distances.

Les sables étudiés ont été prélevés au cours de quatre campagnes réalisées en 1984, 1986 1987, 1988 (Pujos et al. 1989). Ils proviennent (figure 1, tableau I) : 


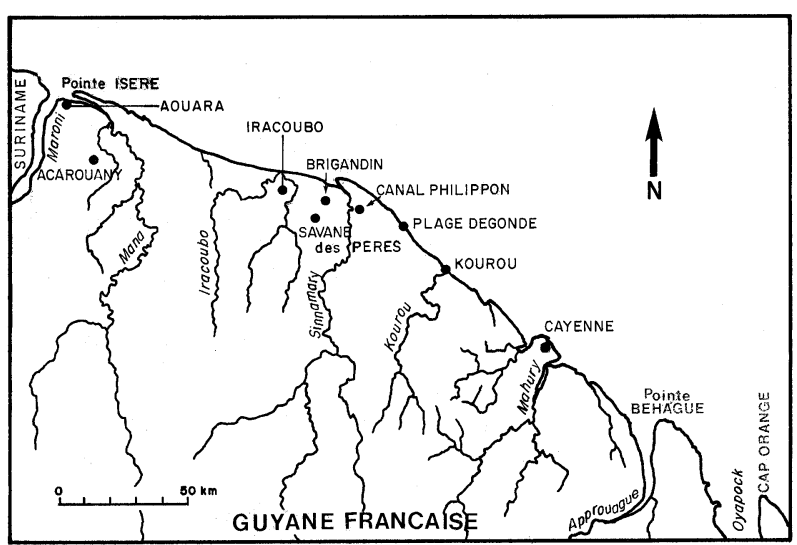

Figure 1. Situation géographique des prélèvements sur les plages et les cordons littoraux de la Guyane française.

Figure 1. Location of the studied area.

- des principales plages de la presqu'île de Cayenne (PS1 à PS9 et 84170), de Kourou (84171), Sinnamary (plage Dégonde, 84173), Aouara (Pointe des Hattes 84185, 84186, 84188, PS10) ;

Tableau I. Plan d'échantillonnage des plages sableuses et des cordons littoraux holocènes de la Guyane française.

Table I. Location of stations (beaches and holocene cheniers).

\begin{tabular}{|c|c|c|}
\hline & $\begin{array}{l}\mathrm{N}^{\mathrm{o}} \\
\text { d'échantillonnage }\end{array}$ & Localisation \\
\hline Plages & $\begin{array}{l}\text { PS1 } \\
\text { PS2 } \\
\text { PS3 } \\
\text { PS4 } \\
\text { PS5 } \\
\text { PS6 } \\
\text { PS7 } \\
\text { PS8 } \\
\text { PS9 } \\
84170 \\
84171 \\
84173 \\
84185 \\
84186 \\
84188 \\
\text { PS10 }\end{array}$ & $\begin{array}{l}\text { Cayenne - Pointe Mahury } \\
\text { Cayenne - Vieux Port } \\
\text { Cayenne - Montjoly } \\
\text { Cayenne - Anse Nadau } \\
\text { Cayenne - Anse de Chaton } \\
\text { Cayenne - Anse de Montabo } \\
\text { Cayenne - Anse de Rémire } \\
\text { Cayenne - Anse de Rémire } \\
\text { Cayenne - Fort Diamant } \\
\text { Cayenne - Pointe St Joseph } \\
\text { Kourou - Les Roches } \\
\text { Plage Degonde } \\
\text { Aouara Les Hattes Bas-estran } \\
\text { Aouara Les Hattes haut-estran } \\
\text { Aouara Les Hattes bas-estran } \\
\text { Aouara Les Hattes }\end{array}$ \\
\hline Cordons littoraux & $\begin{array}{l}84177 \\
84178 \\
84179 \\
84180 \\
84184 \\
84187\end{array}$ & $\begin{array}{l}\text { Savane des Pères } \\
\text { Brigandin } \\
\text { Sinnamary- Canal Philippon } \\
\text { Iracoubo } \\
\text { Acarouany } \\
\text { Aouara Les Hattes }\end{array}$ \\
\hline
\end{tabular}

- de cordons littoraux holocènes issus de la savane des Pères (84177), Brigandin (84178), Sinnamary (84179), Iracoubo (84180), Acarouany (84184), Aouara (Pointe des Hattes 84187).

Les minéraux lourds sont irrégulièrement répartis dans les profils étudiés. Leur comportement, contrôlé par l'hydrodynamique locale (vagues et courants côtiers) dépend de la densité, de la forme et de la taille des différentes espèces minérales (Parfenoff et al., 1970). Une réflexion préalable à l'échantillonnage a donc été nécessaire pour que cette étude reflète le plus fidèlement possible la réalité des cortèges de minéraux lourds.

L'importance de la fraction lourde dans les sédiments sableux est variable, le plus souvent inférieure à $5 \%$ de la totalité des arénites. Ces valeurs sont généralement très supérieures aux moyennes reconnues dans les sables de la plate-forme continentale guyanaise $(M d=0,61 \%$; Pujos et al., 1990). De très fortes concentrations sont localisées dans le moyen estran des plages guyanaises (Germaneau, 1966). À titre d'exemple, une plage de sable beige de la presqu'île de Cayenne (proche du Centre Orstom) montrait un dépôt riche en minéraux lourds dont l'épaisseur moyenne (de l'ordre de 5 à $10 \mathrm{~cm}$ ) décroissait régulièrement de l'amont vers l'aval (tableau II, figure 2). Neuf prélèvements effectués dans cette zone montrent une décroissance de la fraction lourde (par rapport au sédiment décarbonaté) de la côte vers le large. Deux ensembles apparaissent : l'un supérieur (C1 à C6) où la concentration est forte $(M d=76 \%)$, l'autre inférieur $(\mathrm{C} 7$ à $\mathrm{C} 9)$ de concentration beaucoup plus faible $(M d=16 \%)$.

La ségrégation des minéraux lourds par la densité effective sur cette plage résulte de la perte par lessivage des éléments les plus légers : après la séparation magnétique sont isolées, la magnétite $(d=5,17)$, la limonite $(d=3,80)$ et la hornblende $(d=3,17)$. Sur le profil de plage nous observons (figure 2, tableau II) : a) un gradient croissant des teneurs en magnétite avec une concentration maximale dans la partie haute de la laisse à minéraux lourds $(\mathrm{C} 1$ à $\mathrm{C} 6: M d=57 \mathrm{~g})$ et $\mathrm{b})$ un gradient décroissant des teneurs en limonite + hornblende $(\mathrm{C} 1$ à $\mathrm{C} 9: 0,9$ à $8 \mathrm{~g})$.

Sur la plage des Hattes (Aouara) un phénomène comparable se produit avec une accumulation préférentielle des grenats $(d=3,58$ à 4,31$)$ et des staurotides $(d=3,74$ à 3,83 ) en haut de plage. 
Tableau II. Profil de plage dans la presqu'île de Cayenne (proche centre Orstom) Table II. Beach profile (Cayenne peninsula)

\begin{tabular}{lllll}
\hline Gisement & $\begin{array}{l}\text { concentration de la } \\
\text { fraction lourde }(\%)\end{array}$ & limonite + hornblende $(\mathrm{g})$ & magnétite $(\mathrm{g})$ & (limonite + hornblende)/magnétite \\
\hline C1 & 96 & 0,9 & 61 & 0,01 \\
C2 & 94 & 3 & 82 & 0,04 \\
C3 & 94 & 4 & 83 & 0,04 \\
C4 & 66 & 7 & 46 & 0,15 \\
C5 & 50 & 9 & 32 & 0,3 \\
C6 & 58 & 10 & 40 & 0,25 \\
C7 & 25 & 8 & 11 & 0,7 \\
C8 & 13 & 7 & 2 & 5 \\
C9 & 11 & 8 & & 2 \\
\hline
\end{tabular}

Ces résultats sur l'importance et la taille des espèces minérales d'une plage de la Guyane française montrent que :

- les minéraux lourds sont préférentiellement localisés dans les laisses de marée. Leur faible amplitude (Pujos et Froidefond, 1995) et la récurrence des cycles provoquent une accumulation localisée (placer) sur le moyen estran ; - les minéraux à forte densité (magnétite) sont cardinaux dans la partie haute de la laisse alors que les minéraux à plus faible densité (limonite, hornblende) caractérisent la partie basse ;

- la partie centrale de la laisse est la zone privilégiée d'échantillonnage puisque toutes les espèces minérales y sont représentées.

Ces premières observations de terrain conduisent à un échantillonnage raisonné des plages, nécessaire si l'on veut aborder de façon réaliste l'étude des minéraux lourds.

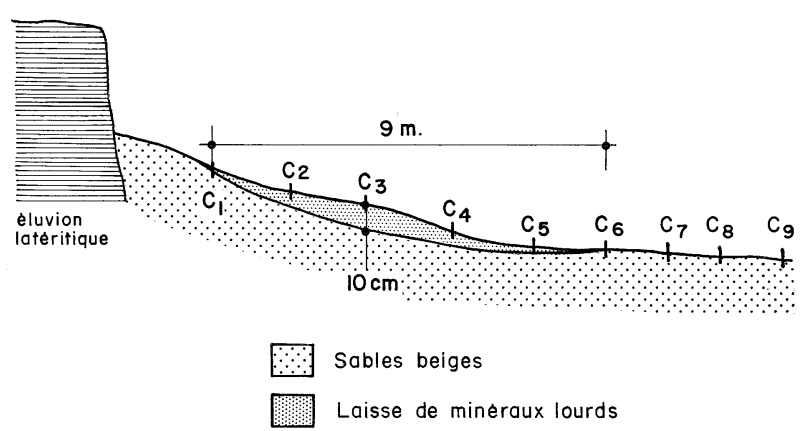

Figure 2. Profil de plage schématique, presqu'île de Cayenne (d'après Germaneau, 1966).

Figure 2. Beach profile, Cayenne peninsula (from Germaneau, 1966).

\section{NATURE DES SABLES}

Les sables, dont la matière organique a été détruite à l'eau oxygénée, ont été tamisés sur une colonne de 16 tamis (série AFNOR) après avoir été lavés et décarbonatés $(\mathrm{HCl})$. Les courbes et paramètres granulométriques consignés dans les rapports de Froidefond (1984) et Pujos et al. (1989) montrent que la plupart des sables de la presqu'île de Cayenne sont fins à grossiers. Leur médiane est comprise entre 205 et $312 \mu \mathrm{m}$ (exception pour les sables de la plage de Montabo : Md PS6 = $395 \mu \mathrm{m})$.

À l'ouest de la presqu'île de Cayenne les sables forment deux grands ensembles : a) très fins à fins, de médiane comprise entre 100 et $200 \mu \mathrm{m}$ (84171, 84173, 84177, 84188 ) et b) moyens et grossiers entre 300 et $400 \mu \mathrm{m}$ (84178, 84179, 84180, 84184, 84186, 84187, PS10), voire très grossiers $(\mathrm{Md} 84185=600 \mu \mathrm{m})$. Les sables sont bien classés à l'ouest, bien à assez classés dans la région de Cayenne.

\section{MÉTHODES D'ÉTUDE}

La fraction du sédiment insoluble à $\mathrm{HCl}$ est essentiellement siliceuse (quartz) mais elle renferme aussi des minéraux lourds dont la densité est généralement comprise entre 3 et 5 alors que celle du quartz est proche de 2,53 . Ces minéraux lourds sont isolés du sédiment brut par deux opérations : a) élimination des carbonates à l'aide d' $\mathrm{HCl}$, rinçage puis séchage à $105^{\circ} \mathrm{C}$, et b) séparation à l'aide de bromoforme $(d=2,89)$ dans des ampoules spéciales puis rinçage à l'alcool méthylique. 
Tableau III. Répartition des principales espèces minérales des sédiments sableux (plages et cordons littoraux) de la Guyane française. Table III. Principal species of heavy minerals (beaches and Holocene cheniers in French Guiana).

\begin{tabular}{|c|c|c|c|c|c|c|c|c|c|c|c|c|}
\hline $\mathrm{N}^{\circ}$ d'échantillon & amphibole & staurotide & épidote & tourmaline & pyroxène & zircon & grenat & andalousite & rutile & biotite & muscovite & sillimanite \\
\hline 84170 & 55 & 17 & 10 & 7 & 8 & 2 & 1 & $<1$ & $<1$ & & & \\
\hline 84171 & 3 & 90 & 1 & 3 & & & & & & 3 & & \\
\hline 84173 & 15 & 2 & & 2 & & 4 & 4 & 1 & & 72 & & \\
\hline 84176 & 8 & 71 & 2 & 15 & & 3 & & & & 1 & & \\
\hline 84177 & & 81 & & 1 & & 1 & & & & & 17 & \\
\hline 84178 & 2 & 85 & 1 & 11 & & & & & & 1 & & \\
\hline 84179 & 3 & 72 & 1 & 9 & & 5 & 10 & & & & & \\
\hline 84180 & 2 & 82 & 2 & 12 & & & 1 & 1 & & & & \\
\hline 84184 & & 35 & & 13 & & 38 & & 13 & 1 & & & \\
\hline 84185 & & 45 & & 12 & & 1 & 34 & 1 & 1 & 5 & & 1 \\
\hline 84186 & 1 & 42 & & 10 & 1 & & 43 & 1 & & 1 & & 1 \\
\hline 84187 & & 27 & & 3 & & $<2$ & 68 & & & & & \\
\hline 84188 & & 29 & & 10 & & & 22 & 1 & 1 & & 37 & \\
\hline
\end{tabular}

Après séchage, le montage est effectué à l'aide d'une résine disposée entre lame et lamelle. À l'issue de cette préparation, effectuée sur la fraction granulométrique $0,500-0,160 \mathrm{~mm}$ qui est la plus représentative, les minéraux lourds sont déterminés au microscope polarisant.

\section{DISTRIBUTION DES PRINCIPALES ESPÈCES MINÉRALES ET ASSOCIATIONS MINÉRALOGIQUES}

Staurotide: ce minéral, caractéristique de toutes les plages et cordons sableux, est cardinal dans la région côtière centrale entre Kourou et Iracoubo $(M d=78 \%)$. À l'ouest (Pointe des Hattes) comme à l'est (presqu'île de Cayenne), les teneurs y sont moindres, respectivement de 36 et $17 \%$.

Grenat almandin : cardinaux dans tous les sites de la Pointe des Hattes. Les teneurs y sont de l'ordre de $70 \%$ sur les cordons, $56 \%$ en haut de plage, $29 \%$ en bas de plage.

Tourmaline : présente dans tous les sites avec des pourcentages moyens de $8,5 \%$ de la région de Cayenne à la savane des Pères, de $10 \%$ plus à l'ouest jusqu'au fleuve Maroni.

Pyroxène: uniquement rencontré dans la région de Cayenne, sous la forme de clinopyroxène.

Amphibole (hornblende verte): une nette décroissance des teneurs en amphiboles apparaît d'est en ouest, 55\% dans la région de Cayenne, 5\% de Cayenne à la savane des Pères, moins de $1 \%$ d'Iracoubo à la Pointe des Hattes.

Épidote (pistachite et zoïsite) et Zircon : ces deux minéraux sont caractéristiques de la presqu'île de Cayenne avec des teneurs de 8 à 10\%.(tableau III.)

Les espèces minérales les plus représentatives du littoral guyanais forment trois cortèges ordonnés d'est en ouest (figure 3) : a) amphiboles dominantes (55\%), staurotides, épidotes et pyroxènes dans la presqu'île de Cayenne (cortège 1) ; b) staurotides largement cardinales $(78 \%)$, tourmalines et amphiboles jusqu'à la Mana (cortège 2) ; c) grenats $(42 \%)$, staurotides $(36 \%)$ et tourmalines $(10 \%)$ de la Mana au Maroni (cortège 3).

Cette configuration générale peut être modifiée avec une augmentation sensible des micas (biotite, muscovite dont l'origine locale est probable) dans des zones de bas estran (plages Degondes et des Hattes). Les estrans peuvent être localement minéralisés avec, à titre d'exemple, de la magnétite voire de l'ilménite dans les laisses de minéraux lourds des plages de la presqu'île de Cayenne (Plages de l'Orstom et du Montabo) et de la pointe des Hattes (Germaneau, 1966 ; Pujos et al., 1990). Sur les cordons situés à l'arrière des plages, magnétite et ilménite ont été reconnues à Aouara, Organabo, Sinnamary (Anonyme, 1980) et à Cayenne dans l'anse de Rémire et Zéphyr (Prost, 1989 ; 1992).

En mer, les dépôts terrigènes (sables grossiers, sables fins et très fins) de la plate-forme continentale renferment, comme ceux du littoral, une quantité significative de 

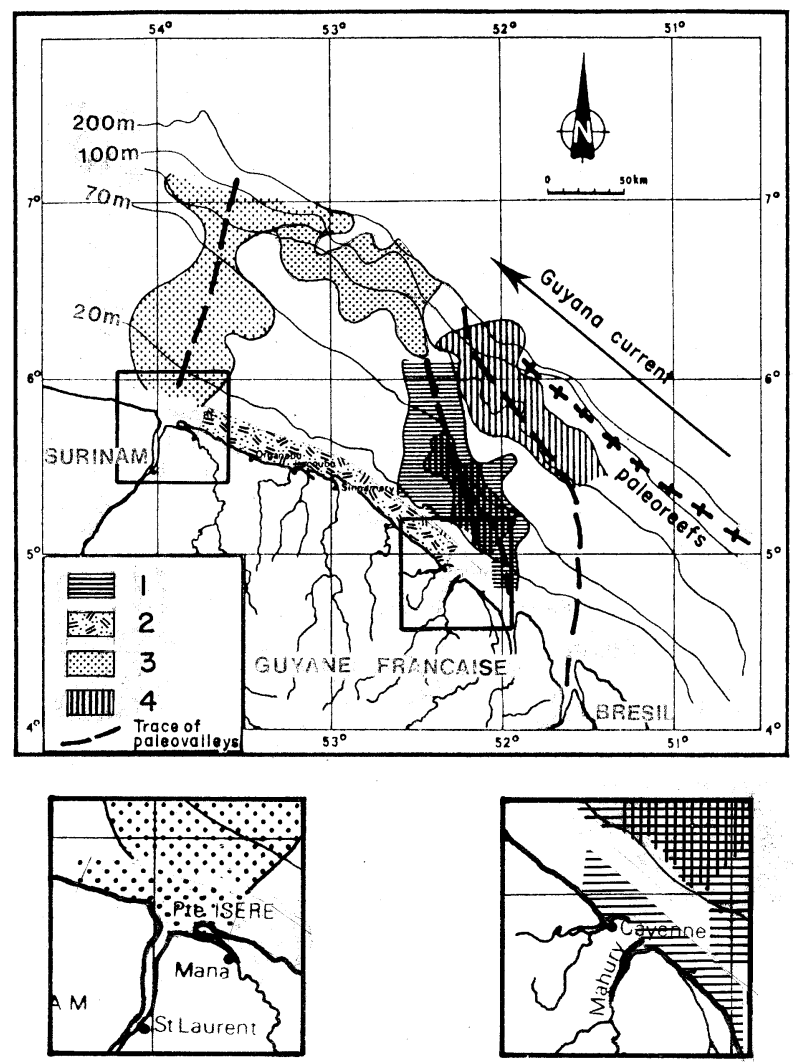

Figure 3. Répartition des cortèges de minéraux lourds (espèces minérales caractéristiques) sur le littoral et sur la plate-forme continentale de la Guyane française : amphiboles et épidotes (cortège 1) ; staurotides (cortège 2) ; staurotides, grenats et tourmalines (cortège 3); amphiboles (cortège 4). Minéraux opaques : magnétite, ilménite.

Figure 3. Heavy minerals on the coast and the continental shelf sediments: Amphibole and epidote (assoc. 1); staurolite (assoc. 2); staurolites, garnet and tourmaline (assoc. 3); amphiboles (assoc. 4).

minéraux lourds. L'étude de leur répartition et de leur origine (Pujos et al., 1990) montre que :

- les amphiboles, toujours présentes, ont des teneurs maximales au large des embouchures des fleuves Oyapock-Approuague-Mahury (à l'est), SinnamaryIracoubo-Organabo (au centre) ;

- les staurotides, également reconnues dans l'ensemble des dépôts, sont cardinales au large du prodelta du Maroni et sur toute la plate-forme externe située à l'ouest du méridien de Kourou ;

- les épidotes et les pyroxènes (au large du Mahury) et les grenats (au large du Maroni) sont spécifiques de ces régions.
Ainsi, trois cortèges minéralogiques sont identifiés sur le littoral, comparables à ceux décrits en mer par Pujos et Odin (1986) puis Pujos et al. (1990) (figure 3): a) amphiboles, épidotes, pyroxènes et tourmalines sur le littoral de la presqu'île de Cayenne et sur la plate-forme continentale au large des fleuves Mahury et Approuague (cortège 1) ; b) staurotides cardinales associées à des amphiboles et des tourmalines sur le littoral de Kourou à la Mana ainsi qu'au large du prodelta du Maroni (cortège $2)$; c) staurotides et grenats dominants, tourmalines, magnétite et ilménite sur les plages et les cordons situés à l'ouest de la Mana ainsi que sur le prodelta du Maroni (cortège 3). Un cortège à amphiboles dominantes est uniquement reconnu en mer de l'Oyapock à Cayenne (cortège 4).

\section{DISCUSSION ET CONCLUSIONS}

\subsection{Les cortèges minéralogiques des grands fleuves guyanais}

Les cortèges minéralogiques des grands fleuves guyanais (Oyapock, Approuague, Mahury, Maroni) ont montré que les minéraux lourds sont représentatifs des formations géologiques traversées (Choubert, 1974 ; Pujos et al., 1990 ; Gibbs et Barron, 1993) (figure 4) :

- grenats et staurotides du Maroni, issus en particulier des schistes, micaschistes et gneiss des séries de Bonidoro et de l'Orapu (synclinorium nord) qui renferment largement ces deux espèces minérales ;

- amphiboles (hornblende), pyroxènes et épidotes (pistachite dominante) de la zone Mahury-Approuague. Cette association caractérise une roche mère basique orthodérivée de métamorphisme faible à moyen que l'on peut rapporter à la série du Paramaca supérieur formée de roches verdâtres à faciès épizonal prépondérant ;

- les minéraux lourds rencontrés dans la basse vallée de l'Oyapock sont plutôt des oxydes de fer variés issus de l'altération climatique de cuirasses latéritiques bien développées sur gneiss et migmatites. Ce cortège montre que le fleuve draine une région constituée de gneiss et de migmatites caraïbes, roches orthodérivées pauvres en silicates d'alumine. Il est complété par des minéraux transparents parmi lesquels les amphiboles sont cardinales, associées à des andalousites et des staurotides. Le cortège de l'Oyapock paraît donc constitué de minéraux opaques issus de l'érosion des latérites superficielles et 


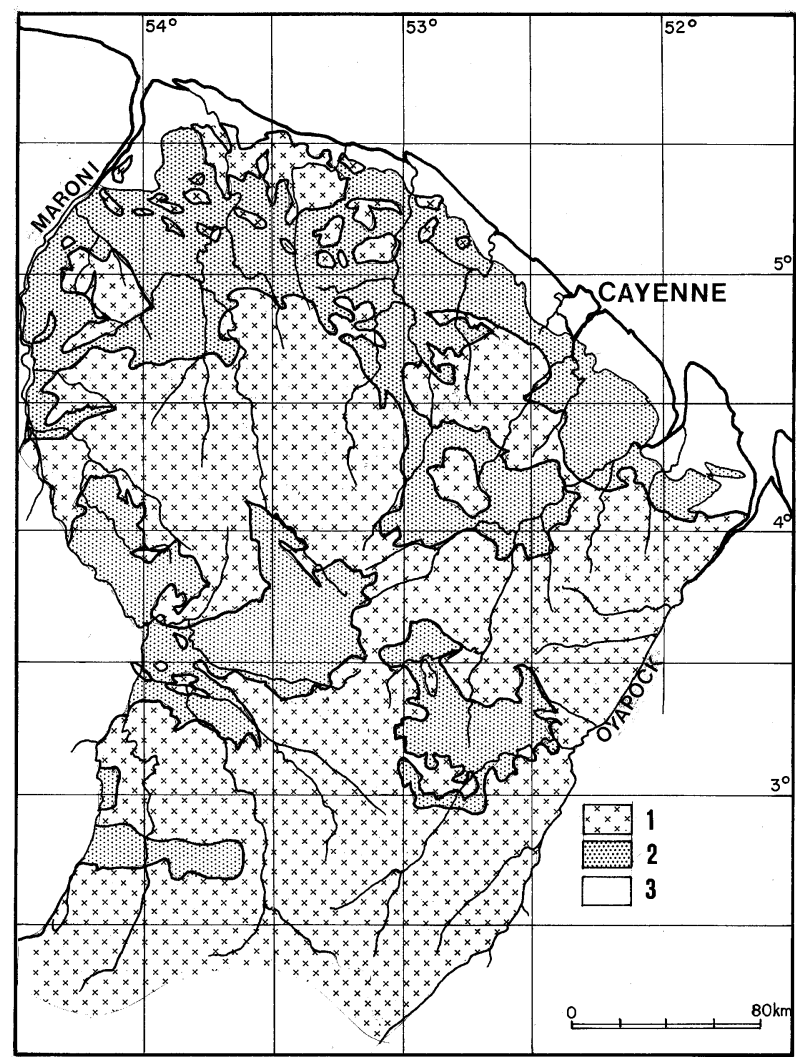

Figure 4. Géologie sommaire de la Guyane française (d'après Choubert, 1974). 1) Granites et gneiss guyanais et caraïbes. 2) Séries métamorphiques de Bonidoro, de l'Orapu et de Paramaca. 3) Alluvions fluviatiles.

Figure 4. Geological map of French Guiana. 1) Guyanese and Caribbean granites and gneiss. 2) Bonidoro, Orapu and Paramaca formations of metamorphic rocks. 3) Fluviatile deposits (from Choubert, 1974).

d'apports paradérivés (silicates d'alumine) et orthodérivés (hornblendes variées) de caractère mésozonal.

Ainsi, chaque bassin versant possède son propre cortège et les cortèges identifiés se retrouvent sur le littoral et sur la plate-forme continentale: staurotides et grenats au large du Maroni et de la Mana d'où seraient originaires les grenats (Krook, 1979), staurotides entre Kourou et la Mana, épidotes et amphiboles au large du Mahury essentiellement, amphiboles dans la partie orientale au large de l'Approuague et de l'Oyapock.

$\mathrm{Si}$ ces cortèges minéralogiques sont caractéristiques de zones géographiques spécifiques du littoral et de la plate-forme continentale, ils ne correspondent pas forcément à des paragenèses directement attribuables à des roches mères affleurantes par suite de mélanges qui se produisent au cours du transport et de la sédimentation : ces cortèges peuvent être définis à partir d'espèces minérales présentant des affinités pétrogénétiques ou paléogéographiques. Comme nous l'avions proposé lors de l'étude des minéraux lourds de la plate-forme continentale (Pujos et al., 1990) nous séparons :

- le cortège des silicates d'alumine purs ou silicate d'alumine associé à $\mathrm{Fe}, \mathrm{Mg}$, (staurotide, grenat) qui appartient à la séquence pélitique (schistes, micaschistes, gneiss). Sur le littoral et en mer, staurotides et grenats caractérisent les apports du Maroni et de la Mana qui drainent des bassins versants constitués des roches précitées. La staurotide (60 à 90\%) est également très représentative de la Sinnamary (Bouysse et al., 1977) ; - le cortège à amphiboles peu colorées et fibreuses associées à l'épidote qui caractérise les apports du Mahury. Cette association appartient à des formations épizonales basiques orthodérivées. Les concentrations les plus fortes sont situées au droit du Mahury et de Kourou ; - le cortège à tourmaline, rutile et zircon. Ces minéraux résiduels, issus d'une longue histoire sédimentaire et très résistants aux agents d'altération physiques et chimiques se rencontrent aussi bien dans les roches magmatiques que métamorphiques de l'arrière-pays guyanais. Ces minéraux constituent le plus souvent un bruit de fond ; lors de fortes concentrations ils ont une signification hydrodynamique ;

- le cortège des minéraux opaques (magnétite, ilménite) et autres composés d'oxydes et d'hydroxydes de fer qui ont surtout pour origine les roches basiques. Ils peuvent cependant être néoformés à la suite de phénomènes pédogénétiques : dans ce cas, les cuirasses latéritiques sur roches acides (granites, gneiss, migmatites) peuvent en fournir une grande quantité. Ce cortège, comme le précédent, ne peut être rattaché à une ou plusieurs roches mères; il constitue un bruit de fond en climat équatorial ; - le cortège de minéraux néoformés (sidérite et verdine) qui a une signification actuelle ou sub-actuelle (Pujos et Odin, 1986).

\subsection{Origine des dépôts}

Trois arguments plaident en faveur d'une provenance locale des espèces minérales lourdes.

1. Il existe une parfaite continuité entre les cortèges de minéraux lourds des roches mères des bassins versants, 
des sables fluviatiles, des plages, des cordons holocènes et de la plate-forme continentale de la Guyane française.

2. Les cortèges minéralogiques gardent sur la plateforme leur orientation originelle. C'est ainsi que :

- les sables à staurotide et grenats des plages, de direction $\mathrm{N} 10 / 20^{\circ}$, matérialisent les paléo-lits du Maroni et de la Mana dont l'existence est confortée par des traces de chenalisation décelées au sondeur $3,5 \mathrm{kHz}$ (Frappa et Pujos, 1994) ;

- sur le continent, la basse vallée de l'Oyapock montre une morphologie d'érosion ravinée sous le remplissage holocène (Boye, 1963).

Ces deux exemples montrent que lors du bas niveau marin correspondant au dernier épisode Glaciaire (Pujos et Odin, 1986), les fleuves guyanais étaient les vecteurs potentiels du matériel détritique.

Les sables à épidote et amphibole de la région MahuryApprouague et les amphiboles de l'Oyapock sont également la trace de systèmes fluviaux.

3. L'étude des différentes fractions granulométriques des flux amazoniens actuels montre la présence : a) de silts grossiers dont la répartition est anarchique : 2,7\% au large de l'Amazone, $6 \%$ au large de l'Oyapock et 1,8\% dans la région de Cayenne; b) de silts moyens qui diminuent régulièrement de l'Amazone (16\%) à l'Oyapock (12\%) et Cayenne $(8,5 \%)$, combinée à une augmentation concomitante des silts fins et des argiles (figure 5). Dans la partie basse des estuaires (Oyapock, Mahury, Maroni), les silts fins et les argiles ont des teneurs comparables à celles rencontrées sur la plate-forme.

Nous constatons que la diminution régulière de la teneur en argiles et en silts fins d'est en ouest traduit le dépôt graduel, par perte de charge, des éléments les plus grossiers des suspensions au cours de leur transit vers l'ouest sous l'action combinée du courant des Guyanes et des houles d'alizés. La grande majorité des argiles et des silts moyens est d'origine amazonienne. Il en est de même pour les vases de la partie basse des estuaires (Pujos et al., 1996). De plus, la répartition désordonnée des silts grossiers suggère plutôt une origine locale par les fleuves. Cette hypothèse est confortée par les travaux de Stuip (1982) qui envisage une telle éventualité pour les silts du Surinam où l'auteur observe une décroissance des teneurs, de l'embouchure de la rivière Surinam vers le large.

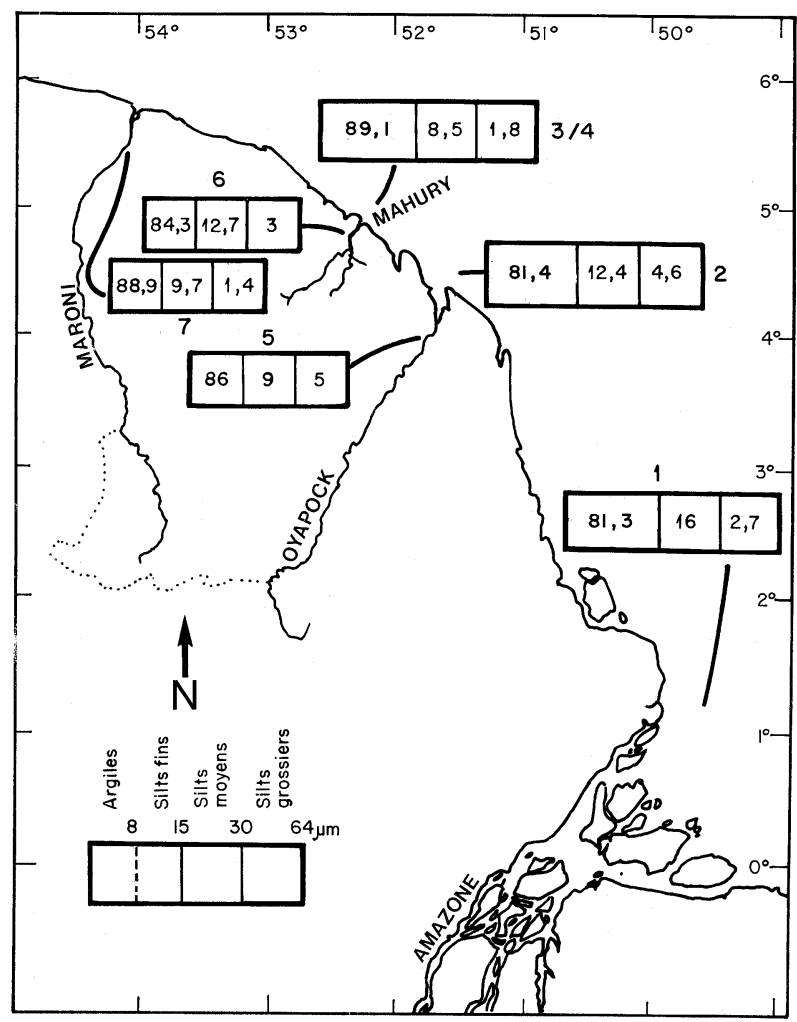

Figure 5. Évolution des fractions granulométriques fines des sédiments de l'Amazone au Maroni. Vasière littorale: Amazone (1); Oyapock (2); partie externe de la vasière de Cayenne (3); banc et inter-banc de Cayenne (4). Estuaires: Oyapock (5); Mahury (6); Maroni (7).

Figure 5. Average distribution of the grain size between the Amazon mouth and the Maroni river. Mud banks: Amazon (1); Oyapock (2); back part of the bank (3); bank and between mudbank (Cayenne) (4); estuaries: Oyapock (5); Mahury (6); Maroni (7).

Les éléments particulaires recueillis sur des filtres montrent que les apports amazoniens transportés en suspension sont constitués d'argiles et de silts fins à moyens. De nos jours, l'apport local paraît donc réel et minoritaire, très vite dilué dans la masse des rejets amazoniens.

Ces trois arguments montrent que les espèces minérales lourdes, et en corollaire les sables dans lesquels elles se trouvent, résultent d'apports locaux. Il ne paraît donc pas nécessaire d'invoquer une origine amazonienne pour certains d'entre eux. 


\section{RÉFÉRENCES}

Anonyme, 1980. Inventaire minier du département de la Guyane française. Bilan et perspectives au 31.12.1979 Ministère de l'Industrie, BRGM, Direction de Cayenne.

Augustinus, P., 1978. The changing shoreline of Surinam (South America), Thesis Utrecht, Natuurwet Studiekring Suriname-Ned. Antillen 95, Utrecht.

Augustinus, P., Hazelhoff, L., Kroon, A., 1989. The chenier coast of Suriname: modern and geological development. Mar. Geol. 90, 269-281.

Boye, M., 1963. La géologie des plaines basses entre Organabo et le Maroni (Guyane française). Mém. Carte Géol. Fr.

Bouysse, P., Kudrass, H., Le Lann, F., 1977. Reconnaissance sédimentologique du plateau continental de la Guyane française (mission Guyamer, 1975). Bull. BRGM 4 (2), 141-179.

Choubert, B., 1974. Le Précambrien des Guyanes. Mém. BRGM 81.

Eisma, D., Van Der Marel, H., 1971. Marine muds along the Guyana coast and their origin from the Amazon basin. Contr. Mineral. Petrol. 31, 321-334.

Frappa, M., Pujos, M., 1994. Late Quaternary evolution of the French Guyana continental shelf evidence from 3.5 Khz data. Mar. Geol. 121, 231-245.

Froidefond, J.M., 1984. Analyse sédimentologique et minéralogique des échantillons prélevés sur les plages de la Guyane(1984) Rapport IGBA.

Froidefond, J.M., Pujos, M., Andre, X., 1988. Migration of mud banks and changing coastline in French Guiana. Mar. Geol. 84, 19-30.

Germaneau, J., 1966. Étude des sables concentrés de Cayenne et de Kourou. Rapport CREO.

Gibbs, A.K., Barron, N.C., 1993. The geology of the Guiana shield. Oxford Monogr. Geol. Geophys., 22. Oxford University Press, Eynsham, UK.

Imbrie, J., Van Andel, T.J., 1964. Vector analysis of heavy mineral data. Contr. Scripps Inst. Oceanogr. 786.

Jones, H., Davies, P., 1979. Preliminary studies of offshore placers deposits, eastern Australia. Mar. Geol. 30, 243-268.

Krook, L., 1969. Investigations on the mineralogical composition of the Tertiary and Quaternary sands in northern Surinam. Verh. Ned. Geol. Mijnb. Genoot. 29, 89-100.

Krook, L., 1979. Sediment petrographical studies in Northern Suriname. Drukkerij Elinkwijk B.V.
Krook, L., 1988. Heavy minerals in Amazon derived sediments on the continental shelf, the Guiana coastal plain and the Guiana marginal plateau. Chapman conference on Amazon dispersal system, Charleston, p. 25.

Krook, L., 1992. Evidence of Amazon provenance of a part of the sandy sediments in the coastal and shelf areas of the Guianas. In : Orstom (Ed.), Symposium PIGC 274. Orstom, Cayenne, pp. 307-326.

Lapierre, F., 1970. Répartition des sédiments sur le plateau continental du golfe de Gascogne. Intérêt des minéraux lourds. Trav. Centre Rech. Et. Oceanogr 10, 1-3.

Parfenoff, A., Pomerol, C., Tourenq, J., 1970. Les minéraux en grains, Méthodes d'étude et détermination. Masson, Paris.

Pomerol, C., 1968. Intérêt et applications de l'étude des minéraux lourds. Rev. Geogr. Phys. Geol. Dyn. 10, 383-396.

Prost, M.-T., 1989. Coastal dynamics and chenier sands in French. Guiana. Mar. Geol. 90, 259-267.

Prost, M.-T., 1992. Sédimentation côtière et formation de cheniers en Guyane : la zone de Cayenne. Évolution des littoraux de Guyane et de la zone caraïbe méridionale pendant le Quaternaire. In : Orstom (Ed.), Symposium PIGC 274. Orstom, Cayenne, pp. 397-414.

Pujos, M., Odin, G.S., 1986. La sédimentation au Quaternaire terminal sur la plate-forme continentale de la Guyane française. Oceanol. Acta 9, 363-382.

Pujos, M., Bobier, C., Chagnaud, M., Fourcassies, C., Froidefond, J.-M., Guillaume, J.-M., Jouanneau, J.-M., Parra, M., Pons, J.-C., Pujos A., de Resseguier, A., Viguier C, 1989. Les caractères de la sédimentation fine sur le littoral de la Guyane française (région de Cayenne) : nature, origine et périodicité de l'envasement. Rapport CORDET, 87001/88310.

Pujos, M., Bouysse, P., Pons, J.-C., 1990. Sources and distribution of heavy minerals in Late Quaternary sediments of the French Guiana continental shelf. Cont. Shelf Res. 10, 59-79.

Pujos, M., Froidefond, J.-M., 1995. Water masses and suspended matter circulation on the French Guiana continental shelf. Cont. Shelf Res. 15, 1157-1171.

Pujos, M., Latouche, C., Maillet, N., 1996. Late Quaternary paleoceanography of the French Guiana continental shelf: clay-mineral evidence. Oceanol. Acta 19, 477-487.

Stuip, J., 1982. Behaviour of silt along the Guyana coast. Proc. FURORIS Congress, pp. 277-328.

Yayer, M.J., 1948. Mission hydrographique de la Guyane française(1936-1937). Ann. hydrogr. 1351, 1-92. 